\section{The Successful Management of a Breast-Feeding Type 1 Diabetic Infant}

\section{Joanna Yuet-ling Tung*, Wai-chong Sat and Wendy Kwan}

Department of Paediatrics and Adolescent Medicine, Queen Mary Hospital, The University of Hong Kong, Hong Kong

\begin{abstract}
Management of infants with type 1 diabetes is challenging to both the families and the medical team. In general, they have unpredictable oral intake and activity level, limited ability to express their needs, and low insulin requirement demanding more precise adjustment. We report a breast-feeding infant with multiple food allergies and type 1 diabetes managed smoothly with insulin pump and continuous glucose monitoring system.
\end{abstract}

Keywords: Breastfeeding; CGMS; CSII pump; Diabetes; Infant

\section{Introduction}

Management of infants with type 1 diabetes is a major challenge to both the families and the medical team. First, this is a rare condition in this age group and hence the experience is scarce. Second, this very young group has their specific age - and development - related characteristics that are distinct from other age groups. In general, they have unpredictable appetite and activity level, and limited ability to express their needs. In addition, due to their small size, their insulin requirement is much lower than older children. This makes precise adjustment of insulin difficult. On one hand, we would like to achieve good glycaemic control to reduce the risk of long - term diabetic complications. On the other hand, strict metabolic control might increase the risk of hypoglycaemia, which, if recurrent, could have adverse effects on neurocognitive outcome [1]; it also could lead to hypoglycemia unawareness and could be especially worrying in this age group. Therefore, good glycaemic control and risk of hypoglycaemia is particularly difficult to balance in this age group.

We report an infant with type 1 diabetes managed smoothly with insulin pump and continuous glucose monitoring system.

*Corresponding author: Joanna Yuet-Ling Tung, Department of Paediatrics and Adolescent Medicine, Queen Mary Hospital, The University of Hong Kong, Hong Kong, Tel: +852 22554485; E-mail: tungylj@hku.hk

Citation: Tung JY, Sat W, Kwan W (2019) The Successful Management of a Breast-Feeding Type 1 Diabetic Infant. J Neonatol Clin Pediatr 6: 032.

Received: May 13, 2019; Accepted: May 30, 2019; Published: June 06, 2019

Copyright: ( 2019 Tung JY, et al. This is an open-access article distributed under the terms of the Creative Commons Attribution License, which permits unrestricted use, distribution, and reproduction in any medium, provided the original author and source are credited.

\section{Case Report}

A 9-month old girl, with good past health except multiple food allergies including cows and soya milk, was admitted for severe diabetic acidosis. The blood glucose was $51 \mathrm{mmol} / \mathrm{L}$ and $\beta$-Hydroxybutyrate (BOHB) was $9.6 \mathrm{mmol} / \mathrm{L}$ on admission, while $\mathrm{pH}$ and bicarbonate were 6.98 and $2.8 \mathrm{mmol} / \mathrm{L}$ respectively. Her antibodies to Glutamic Acid Decarboxylase (anti-GAD), Islet Antigen-2 (IA-2) antibody and anti-islet cell antibody were all positive. She was stabilized with insulin infusion and feeding was resumed after 2 days. She was transited to MDI (Multiple Dose Injection) of subcutaneous insulin with lispro and detemir.

During the transition to subcutaneous insulin, great challenges were encountered. First, she had history of multiple food allergies and was still predominately breast-feeding ad lib before the diagnosis of type 1 diabetes. During her recovery, she refused all kinds of food except direct breast-feeding. In addition, her oral intake was very unpredictable. This makes estimation of carbohydrate intake extremely difficult.

She was put on continuous glucose monitoring (Smartguard ${ }^{\mathrm{TM}}$ Suspend by Sensor), afterwards and the trend of glucose readings with respect to breast-feeding duration and timing of breast-feeding was closely observed. The subcutaneous insulin was titrated accordingly, and a fair glucose profile was achieved.

Subsequently, in addition to continuous glucose monitoring, she was also transited to Subcutaneous Insulin Infusion (CSII) pump therapy (Medtronic MiniMed ${ }^{\circledR}$ 640G System). The daily insulin requirement dropped from 8 to 9 units ( 0.89 to 1 unit $/ \mathrm{kg} /$ day) to 5 to 6 units ( 0.63 to 0.67 units $/ \mathrm{kg} /$ day). The basal insulin ranged from 0.025 to 0.75 units per hour, contributing to around $30 \%$ total daily insulin dose. However, some challenges were noted in the initial phase.

\section{Insulin Sensitivity Factor}

Since her total insulin requirement was very low, her insulin sensitivity factor was around 20 to $25 \mathrm{mmol} / \mathrm{L} / \mathrm{unit}$. However, the pump set-up only allows insulin sensitivity factor to be set between 0.3 to $22.2 \mathrm{mmol} / \mathrm{L} / \mathrm{unit}$. To overcome this, at the period in which the insulin sensitivity factory exceed $22.2 \mathrm{mmol} / \mathrm{L} /$ unit, instead of setting the 'target blood glucose' to be in the normal range, a much higher target was set. In this way, the correction dose would not be over-calculated, even though the insulin sensitivity factor was not exactly her 'real' insulin sensitivity factor.

\section{Very Low Basal Rate}

With such a low total daily insulin dose, her basal insulin rate could be as low as 0.025 unit per hour in certain period of the day. In the Medtronic MiniMed ${ }^{\circledR} 640$ G System, if the basal insulin rate of less than 0.05 units per hour, insulin would only be delivered at pulses at 0 minute and 30 minute of an hour. Therefore, if the pump was transiently disconnected for daily routine e.g. napkin change or showers at those specific times, basically no basal insulin would be delivered, and it would cause glucose level to shoot up. 


\section{Variable Carbohydrate Content and Glycaemic Index in Breast Milk}

Since the infant refused to be fed with bottle but direct breast-feeding, the volume of breast milk could not be accurately determined. Probably partly because of the variations between foremilk and hind milk composition, the carbohydrate content and glycaemic index appeared to vary a lot throughout the day. With close observation of the glucose trend in the CGMS, duration and timing of breast-feeding, we have several observations. First, foremilk has very high glycaemic index and raises glucose very quickly. This was frequently used as a very effective and convenient 'hypoglycaemia treatment' in this patient. Second, if the duration of breast-feeding is long enough and that mother felt that her breast engorgement was completely emptied, the glycaemic index appeared to be much lower and the glucose level would be sustainably high, sometimes requiring the use of square wave bolus to cover for that feeds.

\section{Skin Irritation and Repeated Dislodgement}

In the initial period of using both the insulin pump and CGMS, a lot of skin irritation was observed. This was ameliorated with use of skin prep before application of CGMS sensor and infusion port, followed by application of emollients and topical steroid cream to reddish areas. The dislodgement was also a serious problem with the infusion port almost falling off daily after hot showers. Difference infusion set (MiniMed Sure- ${ }^{\circledR}$ infusion set and MiniMed Mio $^{\circledR}$ infusion set) and sites (thighs and gluteal region) had been tried but the condition remained similar. Eventually, this problem was solved by prior application of skin prep and extra tape on both the sensor and the infusion port.

After around 2-week of adaptation, we finally managed to overcome some of these challenges. A satisfactory glucose profile was achieved (Figure 1). She is currently 2 years of age with normal development and her $\mathrm{A} 1 \mathrm{C}$ remained at $6.5 \%$ to $6.8 \%$. There was no further hospital admission related to her diabetes.

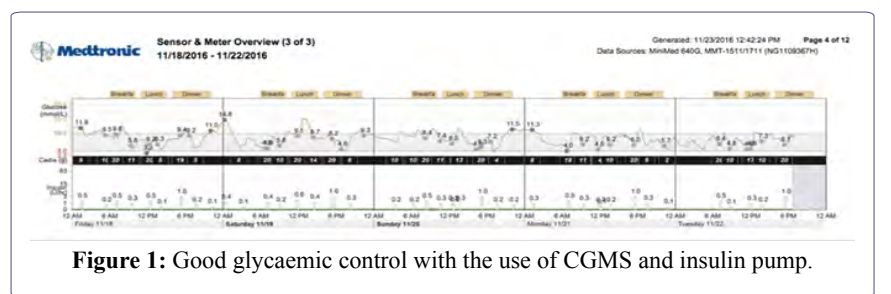

\section{Discussions}

Since diabetes mellitus is less common among young infants, experience in managing this very special group is, in general, scarce. Together with their unique features of variable and unpredictable physical activity and carbohydrate intake, and inability to communicate with their complaints, it makes the management of diabetes particularly challenging. The use of CSII pump integrated with CGMS system might ameliorate the difficulties; yet, the experience in this unique group is also scarce.

In general, using CSII pump therapy required more vigilance especially on the technical aspects and frequent glucose monitoring than basal/bolus insulin injection regimen. However, as reported in qualitative studies, the CSII pump therapy allows more 'freedom, flexibility, and spontaneity in daily lives' [2], 'no longer having to administer painful injections', 'fewer restrictions on the frequency, timing and carbohydrate contents of snacks and meals; and improvements in family life and their child's glycaemic control' [3]. In our case, the use of CSII pump therapy, together with CGMS in this case helped both the parents and the medical team to manage this infant's unpredictable and restricted diet issues smoothly. A smaller pump bolus could be given first before meal, and further doses could be given if she ate more. In this way, even if she refused food, it was no longer an issue as the bolus given prior to meal was only a small bolus. This flexibility relieved many anxieties and potential conflicts on eating between the caretakers and the child. In addition, a more precise insulin dose could be given as bolus (increment as low as 0.025 units) conveniently without insulin dilution. With the suspend before low function, basically our patient had minimal hypoglycaemic episodes, even with such a good HbAlc level. This would not be easily achievable with basal/bolus regime of insulin injection.

In the past decade, the use of CSII pump therapy in children has increased remarkably worldwide, though there is still significant variability among countries in the use of pump technology, contributed by both reimbursement issues and expertise in pump management. There are growing evidences on the safety and better metabolic control with CSII pump therapy when compared to MDI [4-7]. Results from a multi-centered childhood diabetes registry involving more than 16 000 type 1 diabetic children showed that, both HbAlc and daily insulin dose $(\mathrm{U} / \mathrm{kg} / \mathrm{d})$ are lower among children treated with CSII, when compared to basal/bolus regimen, after adjustment for age, gender, and diabetes duration [5]. Similarly, results from three large, transatlantic paediatric registries involving 54,410 children and adolescents also revealed lower mean HbAlc among the pump users than those receiving insulin injection therapy [6]. It has also been reported in a large prospective cohort that, the use of CSII in adolescents is associated with lower rates of microvascular complication, independent of glycemic control or social economic status [8]. To date, there is only one retrospective study on medium term outcome of CSII pump therapy among type 1 diabetic children. It included subjects who used CSII for at least 5 years and reported significantly glycaemic improvement in terms of better A1C 1 year after CSII therapy initiation; with the use of advanced pump features associated with greater improvement in HbAlc. No significant differences were observed for body mass index, insulin requirement or other adverse events and hence it was concluded that its use is safe and effective in the pediatric population [9]. However, long-term data is not available at the moment and its benefits among infants or toddlers are not well described.

Since the insulin dose of infants could be very low, this makes the in-built occlusion detection system unreliable. For example, with the Medtronic MiniMed ${ }^{\circledR}$ 640G System, the occlusion alarm would only be triggered by an average of 2.23 units of missed insulin (standard bolus) or 1.97 units of missed insulin (quick bolus). In an infant whose total daily dose is only 5 units, this alarm system would not detect insulin delivery occlusion. Therefore, it is extremely important to have close blood glucose monitoring, desirably CGMS to prevent DKA related to tubing blockage.

The prediction of insulin requirement with respect of breast-feeding was especially challenging in this case. However, with CGMS and very close communication between the mother and the medical 
team, it is not impossible. We could, of course, suggest changing to elemental formula for her cows and soya milk protein allergy rather than continuing with breast-feeding to minimize all these challenges. Nevertheless, we understand the benefits of breast-feeding and also respected mother's strong wish to continue with breast-feeding. We also believe that we should try our best to facilitate our diabetic children to grow up like other ordinary children as far as possible.

Recently, the closed loop system has been shown to improve glycemic control and reduce nocturnal hypoglycemia in both young people and adults with T1DM [10]. However, the system, similar to our current system, was designed mainly for older children but not infants or toddlers, and hence the aforementioned challenges would still exist at the moment. On the other hand, with the real time CGMS, some parents might be tempted to control their children's blood glucose level very tightly, which could potentially induce unnecessary stress and anxiety. Appropriately coaching and counseling by the medical team is needed to achieve a reasonably good metabolic control without inducing too much unnecessary pressure and frustrations to the families.

In summary, we report an infant with type 1 diabetes managed successfully with CGMS and insulin pump. The integrated system allows a more precise glycaemic control and is safe, more physiological and easier for the insulin administration. The success in management does not just lie on the technology, but also close communications and sharing of experience between the parents and the diabetic team. A multidisciplinary team with special expertise in managing young children with diabetes is essential.

\section{References}

1. Schwartz DD, Wasserman R, Powell PW, Axelrad ME (2014) Neurocognitive outcomes in pediatric diabetes: A developmental perspective. Curr Diab Rep 14: 533.
2. Sullivan-Bolyai S, Knafl K, Tamborlane W, Grey M (2004) Parents' reflections on managing their children's diabetes with insulin pumps. J Nurs Scholarsh 36: 316-323.

3. Rankin D, Harden J, Noyes K, Waugh N, Barnard K, et al. (2015) Parents' experiences of managing their child's diabetes using an insulin pump: A qualitative study. Diabet Med 32: 627-634.

4. Olsen B, Johannesen J, Fredheim S, Svensson J (2015) Danish society for childhood and adolescent diabetes. Insulin pump treatment; increasing prevalence, and predictors for better metabolic outcome in danish children and adolescents with type 1 diabetes. Pediatr Diabetes 16: 256-262.

5. Szypowska A, Schwandt A, Svensson J, Shalitin S, Cardona-Hernandez R, et al. (2016) Insulin pump therapy in children with type 1 diabetes: Analysis of data from the SWEET registry. Pediatr Diabetes 23: 38-45.

6. Sherr JL, Hermann JM, Campbell F, Foster NC, Hofer SE, et al. (2016) Use of insulin pump therapy in children and adolescents with type 1 diabetes and its impact on metabolic control: Comparison of results from three large, transatlantic paediatric registries. Diabetologia 59: 87-91.

7. Levy-Shraga Y, Lerner-Geva L, Modan-Moses D, Graph-Barel C, Mazor-Aronovitch K, et al. (2013) Benefits of continuous subcutaneous insulin infusion (CSII) therapy in preschool children. Exp Clin Endocrinol Diabetes 121: 225-229.

8. Zabeen B, Craig ME, Virk SA, Pryke A, Chan AK, et al. (2016) Insulin Pump Therapy Is Associated with Lower Rates of Retinopathy and Peripheral Nerve Abnormality. PloS One 11: 0153033.

9. Mameli C, Scaramuzza AE, Ho J, Cardona-Hernandez R, Suarez-Ortega $\mathrm{L}$, et al. (2014) A 7-year follow-up retrospective, international, multicenter study of insulin pump therapy in children and adolescents with type 1 diabetes. Acta Diabetol 51: 205-210.

10. Kumareswaran K, Elleri D, Allen JM, Harris J, Xing D, et al. (2011) Meta-analysis of overnight closed-loop randomized studies in children and adults with type 1 diabetes: The Cambridge cohort. Diabetes Sci Technol 5: $1352-1362$. 


\section{Hit \\ HERALD}

Journal of Anesthesia \& Clinical Care

Journal of Addiction \& Addictive Disorders

Advances in Microbiology Research

Advances in Industrial Biotechnology

Journal of Agronomy \& Agricultural Science

Journal of AIDS Clinical Research \& STDs

Journal of Alcoholism, Drug Abuse \& Substance Dependence

Journal of Allergy Disorders \& Therapy

Journal of Alternative, Complementary \& Integrative Medicine

Journal of Alzheimer's \& Neurodegenerative Diseases

Journal of Angiology \& Vascular Surgery

Journal of Animal Research \& Veterinary Science

Archives of Zoological Studies

Archives of Urology

Journal of Atmospheric \& Earth-Sciences

Journal of Aquaculture \& Fisheries

Journal of Biotech Research \& Biochemistry

Journal of Brain \& Neuroscience Research

Journal of Cancer Biology \& Treatment

Journal of Cardiology \& Neurocardiovascular Diseases

Journal of Cell Biology \& Cell Metabolism

Journal of Clinical Dermatology \& Therapy

Journal of Clinical Immunology \& Immunotherapy

Journal of Clinical Studies \& Medical Case Reports

Journal of Community Medicine \& Public Health Care

Current Trends: Medical \& Biological Engineering

Journal of Cytology \& Tissue Biology

Journal of Dentistry: Oral Health \& Cosmesis

Journal of Diabetes \& Metabolic Disorders

Journal of Dairy Research \& Technology

Journal of Emergency Medicine Trauma \& Surgical Care

Journal of Environmental Science: Current Research

Journal of Food Science \& Nutrition

Journal of Forensic, Legal \& Investigative Sciences

Journal of Gastroenterology \& Hepatology Research

Journal of Gerontology \& Geriatric Medicine

Journal of Genetics \& Genomic Sciences

Journal of Hematology, Blood Transfusion \& Disorders

Journal of Human Endocrinology

Journal of Hospice \& Palliative Medical Care

Journal of Internal Medicine \& Primary Healthcare

Journal of Infectious \& Non Infectious Diseases

Journal of Light \& Laser: Current Trends

Journal of Modern Chemical Sciences

Journal of Medicine: Study \& Research

Journal of Nanotechnology: Nanomedicine \& Nanobiotechnology

Journal of Neonatology \& Clinical Pediatrics

Journal of Nephrology \& Renal Therapy

Journal of Non Invasive Vascular Investigation

Journal of Nuclear Medicine, Radiology \& Radiation Therapy

Journal of Obesity \& Weight Loss

Journal of Orthopedic Research \& Physiotherapy

Journal of Otolaryngology, Head \& Neck Surgery

Journal of Protein Research \& Bioinformatics

Journal of Pathology Clinical \& Medical Research

Journal of Pharmacology, Pharmaceutics \& Pharmacovigilance

Journal of Physical Medicine, Rehabilitation \& Disabilities

Journal of Plant Science: Current Research

Journal of Psychiatry, Depression \& Anxiety

Journal of Pulmonary Medicine \& Respiratory Research

Journal of Practical \& Professional Nursing

Journal of Reproductive Medicine, Gynaecology \& Obstetrics

Journal of Stem Cells Research, Development \& Therapy

Journal of Surgery: Current Trends \& Innovations

Journal of Toxicology: Current Research

Journal of Translational Science and Research

Trends in Anatomy \& Physiology

Journal of Vaccines Research \& Vaccination

Journal of Virology \& Antivirals

Archives of Surgery and Surgical Education

Sports Medicine and Injury Care Journal

International Journal of Case Reports and Therapeutic Studies 